\title{
Anterior Segment Imaging - Anterior Chamber Angle Assessment
}

\author{
Lisandro Sakata and Kenji Sakata
}

Glaucoma Service, Centro da Visão, Universidade Federal do Paraná, Brazil

\begin{abstract}
Angle closure is a visually destructive form of glaucoma that accounts for approximately half of the worldwide blindness caused by this disease. Angle-closure glaucoma tends to occur in anatomically pre-disposed eyes, and the evaluation of the anterior segment morphology may help identify eyes at risk of angle closure. Ultrasound biomicroscopy is one of the devices developed for anterior-segment imaging, and it helped to provide better understanding of the mechanisms of angle closure. Recently, optical coherence tomography (OCT) technology became available for evaluating the anterior segment of the eye, enabling rapid non-contact imaging of the anterior chamber. Interestingly, anterior segment OCT (AS-OCT) technology appears to detect more eyes with angle closure compared with gonioscopy, and further studies should address how anterior segment imaging findings should be incorporated into the clinical decision-making process. In summary, this article reviews most of the previously published papers on the use of anterior segment imaging in angle-closure glaucoma and tackles some relevant points for the interpretation of imaging exams in daily clinical practice.
\end{abstract}

\section{Keywords}

Angle closure, angle-closure glaucoma, anterior segment imaging, ultrasound biomicroscopy, optical coherence tomography

Disclosure: The authors have no conflicts of interest to declare.

Received: 17 February 2010 Accepted: 16 April 2010 Citation: European Ophthalmic Review, 2010;4:60-4 DOI: 10.17925/EOR.2010.04.01.60

Correspondence: Lisandro Sakata, Rua Itupava 1428, Alto da XV, Curitiba, PR, Brazil, 80040-000. E: clinicasakata@gmail.com

Primary angle-closure glaucoma is a visually destructive type of glaucoma that accounts for approximately half of the worldwide blindness caused by this disease..$^{1-5}$ The closure of the anterior chamber angle (ACA) seems to be the primary abnormality leading to intraocular pressure (IOP) increase and glaucomatous optic neuropathy. ${ }^{6}$ The contact between the peripheral iris and the trabecular meshwork may impair aqueous humour drainage from the anterior chamber by pre-trabecular mechanisms due to the mechanical obstruction of the functional trabecular mesh-work by the peripheral iris, and/or by abnormalities at the ultrastructural level of the trabecular meshwork due to the prolonged friction/apposition of the iris against the angle wall. ${ }^{7}$ Thus, it is likely that the presence of iris-trabecular contact may represent an important risk factor for the development of this disease. The detection of appositional angle closure (temporary) represents an essential step in the prevention of blindness due to angle-closure glaucoma, as interventions such as iridotomy and iridoplasty might halt the angle closure process, preventing the development of glaucomatous optic neuropathy. However, performing a quick, reliable and reproducible assessment of the ACA may represent a major challenge in daily clinical practice.

The current reference standard for evaluating ACA configuration is indirect gonioscopy, which is an exam that can be easily and quickly performed by properly trained ophthalmologists. Gonioscopy is a inexpensive exam as it does not require sophisticated or supplementary equipment. Gonioscopy enables a direct view of a broad part of the angle and the identification of many anatomical reference points. Most importantly, the dynamic part of this exam permits the differentiation between appositional (temporary) and synechial (permanent) angle closure. However, gonioscopy requires considerable skill and experience, and the ACA assessment may be affected by many variables, such as inadvertent pressure on the anterior chamber and light exposure onto the pupils during the examination. Previous studies have shown that even experienced examiners demonstrated only moderate agreement in determining the angle width. ${ }^{8,9}$

In the face of the difficulties and limitations of the reference standard, other methods have been developed in an attempt to obtain more objective measures of the ACA. Ultrasound biomicroscopy (UBM), Scheimpflug photography and anterior segment optical coherence tomography (AS-OCT) are capable of obtaining high-resolution imaging of the anterior segment, and have the potential to be used for the evaluation of the cornea morphology, ${ }_{10,11}^{10}$ to plan the sizing of phakic intraocular lenses ${ }^{12,13}$ and to assess the morphology of trabeculectomy blebs. ${ }^{14-16}$ Moreover, these tools are able to assess and document the ACA configuration and also to perform objective measures of the ACA. However, to date there have been some limitations in using this new technology in the clinical decisionmaking process. This article will focus on ACA assessment obtained by UBM and AS-OCT.

\section{Ultrasound Biomicroscopy}

UBM exam was presented by Pavlin et al. in 1991 and is a noninvasive exam that obtains high-resolution images of the anterior 
segment of the eye. UBM has a linear high-frequency ultrasonic transducer $(50 \mathrm{MHz})$, which emits sound waves through ocular tissues with a penetration depth of $5.5 \mathrm{~mm}$, and detects their reflection from tissue interfaces. ${ }^{17}$ Thus, UBM is capable of obtaining in vivo crosssectional images of the anterior segment structures of the eye with

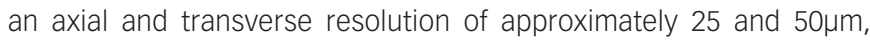
respectively. ${ }^{18,19}$ The scanner produces a $5.0 \times 5.0 \mathrm{~mm}$ field with 256 image lines at a scan rate of eight frames per second. The pixel resolution on the image monitor is about $11-12 \mu \mathrm{m}$ horizontally and about 23-24um vertically.

The UBM evaluation requires immersion techniques. The device probe must be immersed in a saline solution, usually kept in a small and transparent acrylic eye-cup positioned between the eyelids. The exam is usually performed in the supine position, but recent versions of this technology allow the exam to be performed in a sitting position. Nevertheless, an UBM exam may be considered a timeconsuming and relatively unpleasant exam for the patient.

Compared with AS-OCT, UBM image quality may be considered highly dependent on the examiner's skills. For a proper evaluation of the ACA, UBM images must be acquired perpendicularly to the structures of interest. The double arc on the cornea is one of the findings correlated with a properly acquired image (see Figure 1). Of note, the frozen radial section of the drainage angle should aim to include a well-defined scleral spur, which is important for the proper interpretation of the ACA opening.

\section{Anterior Segment Optical Coherence Tomography}

OCT was reported for the first time in 1991, ${ }^{20}$ and it was first used in ophthalmology for imaging the posterior segment of the eye. Since 1994, several modifications have improved the imaging of the anterior segment structures. ${ }^{21-23}$ The wavelength of light used by the AS-OCT was changed to $1,310 \mathrm{~nm}$, which allowed the use of higher-power illumination, which in turn enabled high-speed imaging. ${ }^{12,22-24}$ Thus, the current time-domain AS-OCT is a rapid, non-contact method of obtaining realtime images of the ACA

Analogous to ultrasound, this technology obtains in vivo crosssectional images of tissues, but uses light waves to obtain the reflectivity profile of the structure under investigation. ${ }^{20,25}$ It is important to note that the light used by AS-OCT systems cannot image the anterior segment through the eyelids. Thus, the superior and inferior eyelids must be gently moved out of the way before obtaining AS-OCT scans of the superior and inferior ACA respectively. After acquisition, the scanned images have to be processed by a custom software (de-warping), which compensates for the index of refraction transition at the air-tear interface and the different group indices in air, cornea and aqueous to correct the physical dimensions of the images. ${ }^{12}$

One of the advantages of AS-OCT imaging over UBM is that OCT technology permits a particularly easy non-contact method of imaging the anterior segment and does not require the immersion of the eye in fluid. Additionally, the ability of AS-OCT to image the entire cross-section of the anterior segment in one image frame (see Figure 2) is representative of how remarkable this method is in assessing and documenting the profile of the iris and its relationship to the other anatomical parameters of the eye. However, the image
Figure 1: Ultrasound Biomicroscopy Image of the Anterior Chamber Angle
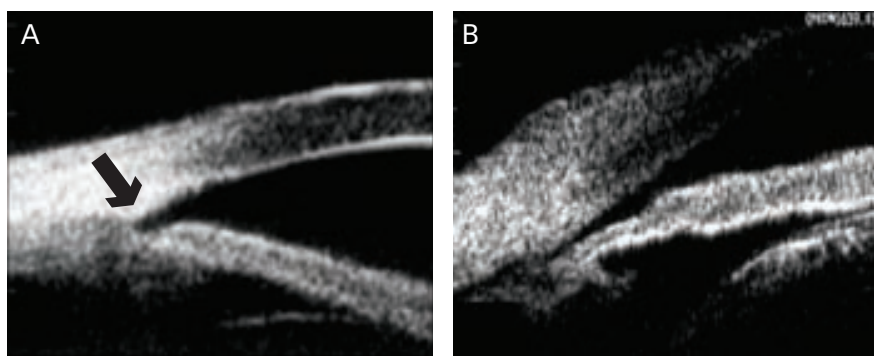

A: The double arc on the cornea indicates that the image was acquired perpendicularly to the structures of interest, and the arrow points out the location of the scleral spur. IB: There is no double arc on the cornea, and the image may not represent the actual morphology of the anterior chamber angle.

\section{Figure 2: Cross-sectional Anterior Segment Optical Coherence Tomography Image of the Entire Anterior Segment of the Eye}

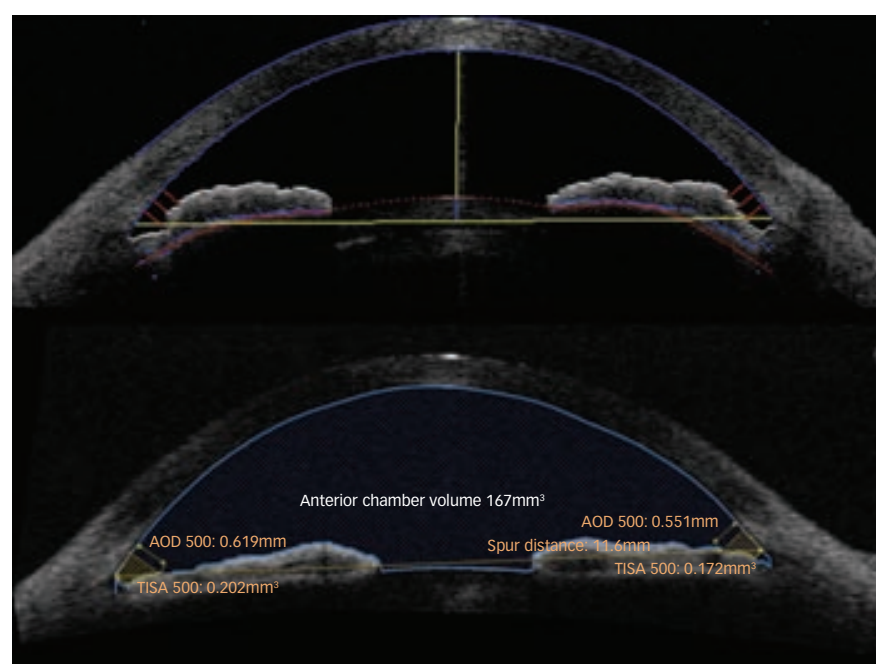

Top image obtained with Visante optical coherence tomography (OCT) and bottom image obtained with slit-lamp-adapted OCT (SL-OCT). The coloured lines represent the anterior chamber measurements evaluated with the semi-automated method using the Zhongshan Angle Assessment Program (ZAAP) and SL-OCT custom software.

resolution in properly acquired UBM images appears subjectively better than that of time-domain AS-OCT images (unpublished data) and the infrared light used by AS-OCT is blocked by the pigment of the iris and the sclera, which precludes the assessment of the structures located behind the iris.

\section{Anatomical Reference Points}

ACA images can be evaluated qualitatively by subjectively determining the open or closed status of the ACA, and quantitatively by objectively measuring ACA parameters. Both analyses are dependent on the recognition of anatomical landmarks, such as the scleral spur location. The scleral spur is usually located on the widest part of the sclera, ${ }^{19}$ appearing as an inward protrusion of the sclera where some of the fibres of the longitudinal bundle of the ciliary muscle attach (see Figure 1). It is an anatomical landmark that reveals the relative location of the trabecular meshwork, which is located approximately 250-500 $\mathrm{mm}$ above the scleral spur along the angle wall. ${ }^{19}$

In qualitative analysis, a closed ACA is defined as the presence of contact between the iris and angle wall above the level of the scleral spur. In quantitative analysis, the exact location of the 


\section{Figure 3: Posterior View of the Human Ciliary Processes} Showing the Variability of their Morphology

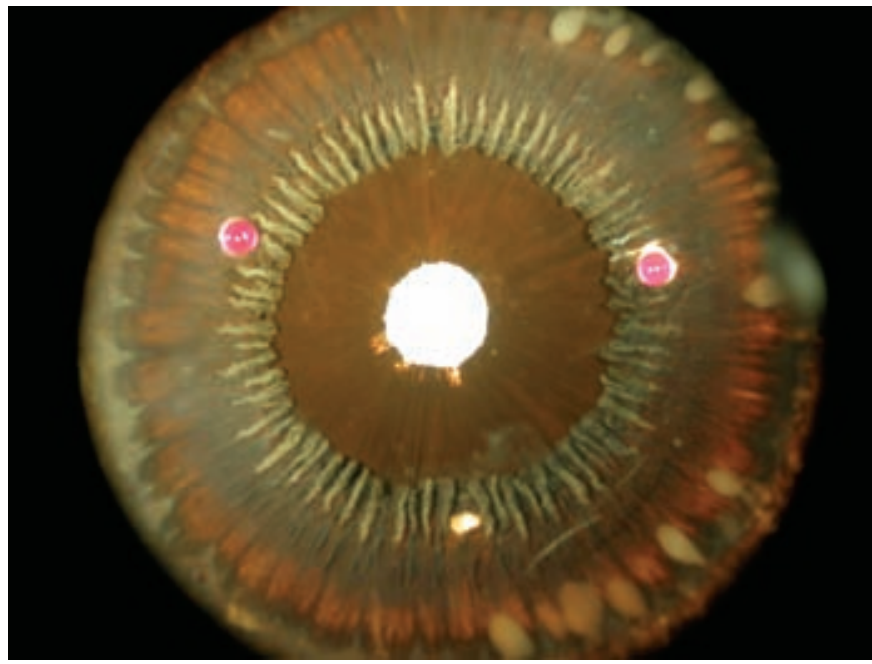

Note the typical ciliary processes and the valley between the processes that can be imaged depending on the location of the cross-sectional scan.

Photograph obtained from the University of Alabama at Birmingham, courtesy of Dr Christopher Girkin and Dr Christine Curcio.

scleral spur is used as the reference point to obtain several measurements of the ACA, such as the angle opening distance (AOD), which is the distance between the angle wall and iris along a perpendicular line to the angle wall located at 500 or $750 \mu$ from the scleral spur (see Figure 2). ${ }^{19}$

Since the location of the scleral spur reveals such relevant information for the proper interpretation of ACA configuration, previous studies evaluated the detectability of this structure in anterior segment imaging. Sakata et al. ${ }^{26}$ observed that the scleral spur location could not be identified in almost 30\% of the AS-OCT images obtained with Visante-OCT (Carl-Zeiss Meditech, Dublin, CA, US - model 1,000, software version 1.0), and the difficulty was greater in eyes with narrow angles and in images obtained in the superior and inferior quadrants. Similar findings were observed with slit-lampadapted OCT (SL-OCT; Heildelberg Engineering, Germany - software version 1.1) images in another study. ${ }^{27}$ With regard to UBM images, no study with a reasonable sample size has directly addressed this issue, although it is possible that the better resolution of UBM images may facilitate the detection of the scleral spur.

However, it is interesting to note that despite the relatively high rate of images in which the exact location of the scleral spur could not be determined, almost $90 \%$ of the AS-OCT images could still be graded qualitatively by glaucoma specialists as having either an open or closed ACA (qualitative analysis). ${ }^{26-28}$ According to the authors, this was possible by relying on other morphological landmarks, such as the insertion of the iris and the peripheral iris profile. Nevertheless, this problem of determining the scleral spur location may hamper quantitative analysis of ACA parameters in a substantial proportion of subjects, ${ }^{26}$ therefore precluding the use of new anterior segment parameters that seem to improve the diagnostic performance of imaging devices in detecting eyes at risk for angle-closure. However, as technology advances, updates to current software and hardware may improve image resolution, enhancing the detection of the scleral spur and possibly new anatomical reference points.

\section{Reproducibility}

Previous studies evaluated the reproducibility of ACA measurements in UBM and AS-OCT images. Measurement variability can be influenced by variability in image acquisition, and also by image analysis due to the variability in subjectively determining anatomical landmarks such as the location of the scleral spur. Thus, measurement variability represents an important step in the validation process of a new technology.

Only a few studies have evaluated the reproducibility of measuring ACA parameters with UBM..$^{29,30}$ Both studies observed a good reproducibility of anterior segment measurements in UBM images; however, both only evaluated eyes with open ACA. The reproducibility of angle measurements in eyes with a narrow angle was not assessed and this may not be as good as in open angles, as the location of the scleral spur seems to be less detectable in narrow angles. ${ }^{26,31}$

In terms of the reproducibility of measurement in ACA parameters with AS-OCT, some papers have evaluated prototype versions of the devices. ${ }^{32,33}$ Recently, Leung et al. ${ }^{34}$ evaluated the agreement and inter-observer reproducibility of angle measurements obtained with Visante and SL-OCT at the nasal and temporal angles of 49 eyes of healthy normal subjects. The study observed that both AS-OCT devices demonstrated good inter-observer reproducibility for angle measurements. However, most of the participants included in this study had open angles on gonioscopy, and as mentioned previously, the reproducibility of ACA measurements in eyes with narrow angles may not be as good as in open angles. In fact, console et al. ${ }^{35}$ evaluated the reproducibility of the commercial available visante-OCT in eyes with open and also narrow ACA using a specific software (Zhongshan Angle Assessment Program [ZAAP], Guangzhou, China). The authors observed that reproducibility was worse in eyes with narrow ACA and the determination of the scleral spur location by different examiners was an important source of variability. ${ }^{35}$

\section{Recent Papers on Quantitative Analysis of Anterior Segment Optical Coherence Tomography}

As mentioned above, AS-OCT is capable of imaging the entire crosssection of the anterior segment in one image frame. This characteristic has enabled the measurement of novel anterior chamber parameters, such as iris morphology and global anterior chamber indices, including anterior chamber volume, anterior chamber area, lens vault and anterior chamber width.

Some recent papers have investigated these novel parameters. Wang et al. ${ }^{36}$ observed that iris thickness, curvature and area are independently associated with narrow angles, particularly in women and older subjects. Other papers presented at the Association for Research in Vision and Ophthalmology (ARVO) and the American Academy of Ophthalmology (AAO) observed that anterior-chamber width was also independently associated with narrow angles. Moreover, the anterior-chamber volume was one of the best diagnostic indicators in detecting eyes at risk for angle closure. Although the number of eyes that had to be excluded from analysis due to poor location of the scleral spur, novel software may become capable of measuring global anterior chamber indices, such as anterior chamber volume, independently of the location of the scleral spur. 
Table 1: Number of Quadrants with Closed Anterior Chamber Angle on Gonioscopy and Anterior Segment Optical Coherence Tomography Imaging (Visante-OCT) in Three Studies

\begin{tabular}{|c|c|c|c|c|c|c|}
\hline \multirow[t]{3}{*}{ Quadrant } & \multicolumn{2}{|c|}{ Nolan et al., $2007^{40}$} & \multicolumn{2}{|c|}{ Sakata et al., $2008^{28}$} & \multicolumn{2}{|c|}{ Sakata et al., $2009^{27}$} \\
\hline & Closed on & Closed on & Closed on & Closed on & Closed on & Closed on \\
\hline & AS-OCT (\%) & Gonioscopy & AS-OCT & Gonioscopy & AS-OCT & Gonioscopy \\
\hline Superior & NA & NA & 48 & 29 & 54 & 35 \\
\hline Inferior & 68 & 41 & 43 & 22 & 58 & 28 \\
\hline Nasal & 40 & 16 & 18 & 14 & 28 & 26 \\
\hline Temporal & 31 & 18 & 12 & 20 & 19 & 26 \\
\hline Total & 67 & 44 & 30 & 21 & 40 & 29 \\
\hline
\end{tabular}

AS-OCT $=$ anterior segment optical coherence tomography; NA = not available.

\section{Comparison of Anterior Segment Optical Coherence Tomography and Ultrasound Biomicroscopy}

Dada et al. ${ }^{37}$ evaluated 63 subjects with normal eyes, and observed a good correlation between AS-OCT and UBM measurements obtained at the nasal and temporal quadrants. However, the comparison between the devices in eyes with narrow ACA remains to be evaluated

Radhakrishnan et al..$^{15}$ compared the quantitative measurements provided by UBM and a prototype version of Visante-OCT in a small sample of 17 normal subjects and seven subjects with a narrow ACA, as determined by gonioscopy. The authors observed that both methods had a similar discriminatory power to detect eyes with narrow ACA. They also observed that there were significant differences between some ACA parameters measured by the two devices (angle recess area at 500 and $750 \mu \mathrm{m}$, trabecular-iris space area at $750 \mu \mathrm{m})$, as UBM tended to show smaller measurements.

There are only a few studies comparing UBM and AS-OCT, and they have either a small sample size and/or selective inclusion/exclusion criteria and/or only evaluate eyes with open ACA. Further studies comparing these two techniques are needed.

\section{Comparison of Time-domain Anterior Segment Optical Coherence Tomography Devices}

Two studies have compared the measurements obtained with the two time-domain AS-OCT (Visante-OCT and SL-OCT). Leung et al. ${ }^{34}$ found poor agreement between the measurements obtained with the two devices at the nasal and temporal angles of 49 eyes of healthy normal subjects. Sakata et al. ${ }^{27}$ compared the AS-OCT measurements obtained in a hospital-based sample of subjects with open and narrow ACA, and observed that the measurements obtained with the two devices were not interchangeable. Differences in imaging acquisition (variation in the state of accommodation, exact scan location, scanning speed and light conditions) and in imaging processing (algorithms for image 'de-warping' and resolution of the scans) between Visante-OCT and SL-OCT in both studies may explain this poor agreement. Clinicians should be aware of the potential differences in angle measurements obtained with these instruments.

\section{Comparison of Imaging Exams and Gonioscopy}

Barkana et al. ${ }^{38}$ compared the agreement between gonioscopy and UBM imaging in detecting appositional angle closure in 17 eyes with narrow ACA. The authors of this study concluded that there was a high agreement between methods, and performing gonioscopy in a completely dark room is important in order to avoid

\section{Figure 4: Cross-sectional (Not 'De-warped') High-definition Optical Coherence Tomography Image of the Angle}

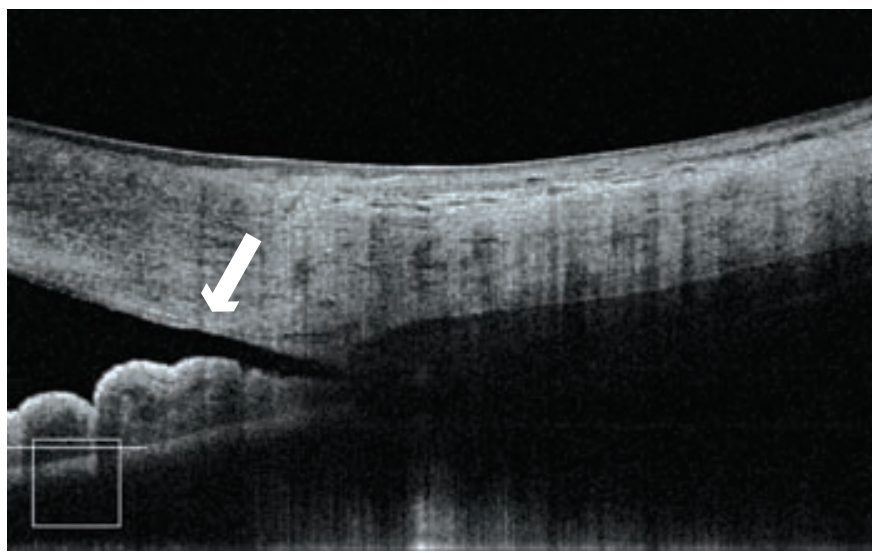

The image shows the termination of the Descemet membrane (Schwalbe Line - white arrow) and also the Schlemm's canal.

Photograph courtesy of Dr Tin Aung, Singapore Eye Research Institute.

misdiagnosis of eyes at risk of angle closure. Of note, the study sample was rather small and included selected patients with narrow ACA only. It is possible that the good agreement between the methods may not be observed in population less prone to bias, such as a community- or population-based study. Indeed, even in hospital-based studies, such as the one performed by spaeth et al. in 22 patients with varied angle width, some discrepancies in appositional angle closure were detected by gonioscopy and UBM. ${ }^{39}$

Nolan et al. ${ }^{40}$ compared the performance of a prototype version of the Visante-OCT and gonioscopy in detecting closed ACA. The authors observed that AS-OCT detected more closed ACA than gonioscopy. They have hypothesised that the disagreement between these two techniques may be partially explained by the fact that while AS-OCT uses infrared light and does not require contact with the eye, inadvertent indentation by the gonio-lenses and the use of light during gonioscopy may artificially open the ACA. In addition, Nolan et al. ${ }^{24}$ hypothesised that the anatomical landmarks considered in the two techniques may not be the same. A closed ACA in gonioscopy was determined by the observation of contact between the iris and the posterior trabecular meshwork; however, in imaging any contact between the iris and angle wall anterior to the scleral spur would suffice to consider an ACA as closed. $28,31,40$

Two further studies from the same group, but more importantly using the results of two different gonioscopists, have confirmed the 
findings of Nolan et al..$^{40}$ Using the commercially available Visante-OCT and SL-OCT, these studies observed that AS-OCT imaging detects more closed ACA than gonioscopy, mainly on the superior and inferior quadrants (see Table 1). ${ }^{27,28}$ The authors of these studies have observed that a minority of eyes may show the opposite: a closed ACA on gonioscopy and an open ACA on imaging. These findings were observed in eyes with a pronounced convexity of the iris ('over the hill' configuration), in which the gonioscopist could not observe the posterior trabecular meshwork even with a slight tilt of the goniolens, but imaging could clarify that, although narrow, there was no irido-trabecular contact.

\section{Peculiarities in Interpreting Anterior Chamber Angle Images}

As both UBM and AS-OCT acquire cross-sectional images of the ACA it is important to note that these images represent a frozen radial section of the drainage angle. These images only reflect the ACA morphology of the scanned region of the angle, and in some cases this region may not be representative of the ACA morphology of the remaining portions of the ACA.

The morphology and position of the ciliary processes observed on UBM images may vary in different radial scans (through a typical ciliary process or the valley between ciliary processes) ${ }^{41}$ (see Figure 3). Slight differences in the acquisition location of radial scans may influence the interpretation of the results, and it is recommended that examiners should perhaps attempt to obtain radial sections passing through a typical ciliary process (probably better than obtaining scans randomly). Sakata and Malta ${ }^{42}$ showed that the image acquisition technique may affect the diagnosis of the plateau iris mechanism, as the absence of ciliary sulcus per se should not determine plateau iris diagnosis.

Within the same rationale, eyes with an isolated peripheral anterior synachiae may have a similar confounding effect with both UBM and AS-OCT. A radial scan through a peripheral anterior synachiae would show a closed angle, whereas a radial scan obtained just next to it (with no peripheral anterior synachiae) could show a wider angle. Thus, for a proper interpretation of the ACA configuration, imaging results should always be correlated with gonioscopy findings.

\section{Interpreting Anterior Chamber Angle Image Findings in Clinical Practice}

Before becoming widely accepted in clinical practice, new technologies have to be validated against existing standards. However, potential limitations of the reference standard technique may affect the evaluation of the new technology. Gonioscopy has inherent limitations, as it is an exam that requires contact of the goniolens with the eye, and it needs visible light to visualise the ACA structures. Thus, the examining conditions of gonioscopy and imaging exams are simply not the same.

Currently, it is not known whether anterior segment imaging is actually detecting eyes at risk of angle closure that are being missed by gonioscopy, or (for some reason) is overdetecting closed ACA (falsepositive cases). It seems that peculiarities in the methods of assessing and interpreting the ACA configuration of each technique may account for at least some of the discrepancies between gonioscopy and imaging exams. Imaging results alone should not guide clinical decisions, particularly when determining the need for treatment modalities such as iridoplasty or early cataract extraction. Nevertheless, the technology is advancing rapidly, and new devices have the potential to considerably improve anterior segment image resolution (see Figure 4$)^{43}$ and, consequently, the interpretation of its results.

In conclusion, longitudinal studies are required to clarify how anterior segment imaging findings should be incorporated into the clinical decision-making process. Until then, it is important to emphasise that gonioscopy is a required feature of an eye examination, and a properly performed dark-room gonioscopy is the reference standard exam for assessing ACA.

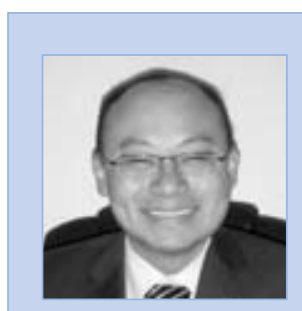

Lisandro Sakata works in the Glaucoma Service in the Department of Ophthalmology at the Universidade Federal do Parana (UFPR), Brazil. His research interests focus on angle closure, methods of anterior segment imaging, glaucoma epidemiology and visual function in glaucoma. Dr Sakata obtained his MD and completed his ophthalmology residency at UFPR. Dr Sakata completed a fellowship in glaucoma and obtained his $\mathrm{PhD}$ at the Universidade Sao Paulo. He also completed two fellowships in glaucoma, one at the University of Alabama, Birmingham, and the other at the Singapore Eye Research Institute.

\footnotetext{
Foster PJ, et al., Arch Ophthalmol, 1996;114:1235-41. Foster PJ, et al., Arch Ophthalmol, 2000;118:1105-11. Dandona L, et al., Ophthalmology, 2000;107:1710-16. Foster PJ, et al., Br J Ophthalmol, 2001;85:1277-82 Quigley HA, et al., Br J Ophthalmol, 2006;90:262-7. Foster PJ, et al., Br J Ophthalmol, 2002;86:238-42. Sihota R, et al., Indian I Ophthalmol, 2001;49:255-9. Nolan WP, et al., Br J Ophthalmol, 2000;84:1255-9. Foster PJ, et al., Br J Ophthalmol, 2000;84:186-92.

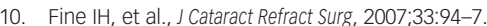

11. Lai MM, et al., J Cataract Refract Surg, 2006;32:1860-65.

12. Goldsmith JA, et al., Ophthalmology, 2005;112:238-44.

13. Baikoff G, J Cataract Refract Surg, 2006;32:1827-35.

14. Singh $\mathrm{M}$, et al., Ophthalmology, 2007;114(1)4:47-53.

15. Leung CK, et al., Br J Ophthalmol, 2007;91(3):340-44.

16. Muller M, et al., Curr Eye Res, 2006:31:909-15.
}

17. Nolan W, Curr Opin Ophthalmol, 2008;19:115-21.

18. Pavlin CJ, Can J Ophthalmol, 1995:30:225-9.

19. Pavlin CJ, et al., New York: Springer-Verlag, 1994.

20. Huang D, et al., Science, 1991;254:1178-81.

21. Izatt JA, et al., Arch Ophthalmol, 1994;112:1584-9.

22. Hoerauf H, et al., Ophthalmic Surg Lasers, 2000;31:218-22.

23. Radhakrishnan $\mathrm{S}$, et al., Arch Ophthalmol, 2001:119:1179-85

24. Radhakrishnan S, et al., Arch Ophthalmol, 2005;123:1053-9.

25. van Velthoven ME, et al., Prog Retin Eye Res, 2007;26:57-77.

26. Sakata LM, et al., Arch Ophthalmol, 2008;126:181-5.

27. Sakata LM, et al., Eye, 2010;24(4):578-87.

28. Sakata LM, et al., Ophthalmology, 2008;115:769-74.

29. Tello C, et al., Invest Ophthalmol Vis Sci, 1994;35:3549-52.

30. Souza Filho EC, et al., Ara Bras Oftalmol, 2005;68:177-83.
31. Nolan W, Br J Ophthalmol, 2008;92:1575-6

32. Muller M, et al., J Cataract Refract Surg, 2006;32:1803-8.

33. Radhakrishnan $\mathrm{S}$, et al., Invest Ophthalmol Vis Sci, 2007:48:3683-8.

34. Leung CK, et al., Invest Ophthalmol Vis Sci, 2008:49:3469-74.

35. Console JW, et al., Br J Ophthalmol, 2008;92:1612-16.

36. Wang B, et al., Ophthalmology, 2010;117:11-17.

37. Dada $T$, et al., J Cataract Refract Surg, 2007:33:837-40

38. Barkana Y, et al., Arch Ophthalmol, 2007;125:1331-5.

39. Spaeth GL, et al., Trans Am Ophthalmol Soc, 1995;93:337-47, discussion 347-51.

40. Nolan WP, et al., Ophthalmology, 2007;114:33-9

41. Pavlin CJ, et al., Am J Ophthalmol, 1992:113:390-95

42. Sakata LM, et al., J Glaucoma, 2006;15:371-9.

43. Wong HT, et al., Arch Ophthalmol, 2009;127:256-0 